\title{
VCL Construction and Optimization Based on Vmware Private Cloud
}

\author{
Qinghua Sun ${ }^{1, a}$, Jianhai $\mathrm{Yu}^{2, \mathrm{~b}}$ \\ ${ }^{1.2}$ Electrical and Mechanical College of Beijing Union University, Beijing, China, 100020 \\ a jdtqinghua@buu.edu.cn
}

\begin{abstract}
Keywords: Cloud Computing Virtualization Technology, Vmware Private Cloud, Virtual Computer $\mathrm{Lab}(\mathrm{VCL})$
\end{abstract}

\begin{abstract}
In this paper, the characteristics of computer practice teaching universities and research requirements, analysis of cloud computing and virtualization technology, noted that the use of virtualization technology on campus to build cloud-based platform for virtual computer lab (Virtual Computer Laboratory) to meet the campus computer services demand, is an effective way and reasonable option. Comparing the calculated deployment and mainstream virtualization platform in the cloud, build private cloud recommended Vmware VCL programs and made a number of recommendations to build points and performance optimization.
\end{abstract}

\section{Introduction}

In 2010, the "Long-term Education Reform and Development Plan (2010-2020)" education information technology for the first time included in the national development strategies. In recent years, colleges and universities information construction has made considerable progress, university computer information center and a physical computer lab (room) Construction of replacement significantly faster.

The college physical computer lab of the author bears a lot of computer experiment teaching, training and research projects. Room construction as a measure of the level of university school quality an important part, but there are also high construction investment and maintenance costs, management and maintenance workload, low efficiency and limited time and space problems [1]. With the construction of our hospital campus network development, the network has covered classrooms, libraries, student dormitories throughout campus. Cloud computing makes virtual computer lab (Virtual Computer Laboratory) based implementation possible, even more importantly, it also provides for the construction of university computer lab, a new way of thinking. According to estimates, the use of cloud computing technology to build virtual computer lab platform than traditional physical room (about 50 people meet on board) can save about two-thirds of the investment. And energy consumption for everyday use is also greatly reduced.

A cloud computing platform in existing computer resources (mainly existing server resources), based on adding the appropriate hardware and software cloud system is built, through the campus network interconnection of existing computing resources to integrate in order to achieve at any time anywhere and get the same physical room environment and computer skills. It can be concluded that the use of technology to build VCL cloud computing platform is the next room construction as well as information technology education development.

\section{Cloud Computing and Virtualization Technology}

Cloud computing refers to the network connecting a large number of computing resources unified management and scheduling, constitute a vast pool of resources (networks, servers, storage, applications, and services) to provide users with on-demand service based. Therefore, cloud computing can also be called cloud services, is a new IT service model. From the customer service side is to see: In the cloud computing environment, users do not need self-built base system can focus on their business. Users can obtain needed resources on the network, according to the amount paid. From the cloud computing services provided look back: cloud computing resources to achieve centralized, large-scale, we can make better use of resources and reduce costs. 
At present, the type of cloud service providers fall into three categories: IaaS (infrastructure as a service), PaaS (Platform as a Service), SaaS (software as a service). Obviously, VCL cloud computing needs after the main construction platform can provide both services. Mode can also be deployed from the cloud into three categories: public, private and hybrid clouds. Public cloud means cloud service providers through its own cloud infrastructure to provide services directly to users, users can access the service through the Internet, but does not have the resources to cloud computing. Public cloud downside is that there are some data security risks. Private cloud inside the units deployed in the user, the data security, system availability can be controlled by their own, but the lack of this model is the larger initial investment. In a particular embodiment of the process, we can continue to integrate the use of existing resources and gradually improve, in order to reduce the initial investment. Hybrid cloud is between a model between public and private clouds, which both own cloud computing infrastructure, while also using the service provided by an external public cloud.

Virtualization technology is the core and foundation of cloud computing, quality virtualization technology is the key to cloud computing applications. Virtualization includes server virtualization, storage virtualization, desktop virtualization, and application virtualization. The first two techniques can build a "cloud" that is a virtual resource pool and the latter two technologies provide users using the platform and program applications.

The first virtual computer lab (VCL) is a North Carolina State University (NCSU) and IBM co-founded, the use of virtualization technologies from IBM blade servers and laboratory PC via cloud computing, for teachers and students with easy to expand the use of resources, high-performance platform [2] [3].

\section{Private Cloud Platform Construction VCL Based on Vmware}

The Option of Vmware Private Cloud. Comparison of three cloud deployment models, from the current actual situation of individual institutions, the authors believe that the use of private cloud model of the engine room transformation management is a viable option. Private cloud computing platform construction program is more mature virtualization technology. It can be achieved through virtualization hosts and storage resources integration and sharing integrated utilization, which not only improve resource utilization, reduce costs, but also help reduce the complexity of management. VMware, Microsoft and Citrix virtualization vendors are now main stream and it is recommended to build VCL uses an absolute leadership position in the virtualization services, as a mature and stable performance VmwareVSphere cloud platform.

Server Virtualization. Server virtualization means to integrate the hardware resources of one or more servers, in the above use virtual machine technology, create one or more virtual servers, unified deployment of the physical hardware resources to achieve efficient use of hardware. Server virtualization is cloud computing an important part. If a server configuration is good, there are WEB services and mail services, FTP services and some other services need to run, under the existing conditions if all deployed on a single server and hardware resources to meet the requirements, but it is easy to see the mutual interference caused by service stability will be substantially reduced. In this case if the first deployment of a number of virtual servers on the server, and then deploy a single service in each virtual server, so ease of use and stability is greatly improved. Another example has three general performance of the server, you need to run two large service, consuming resources, a single server cannot meet the requirements, then you can combine the three server hardware resources using unified deployment of virtualization technology, the formation of pool of hardware resources and then use these resources to build two virtual servers, each running two services, so to meet the hardware requirements, and secondly, independent of each other. In fact, large-scale applications, often to dozens or even hundreds of servers such hardware resource pools, such as 100 servers, resource pools consisting of the deployment of at least 500 or more virtual servers. Because each server is not possible to stay in a $100 \%$ hardware footprint, so idle resources can be dynamically allocated to other virtual server resource pool needed to greatly reduce hardware costs, improve management efficiency. 
Desktop Virtualization. Desktop virtualization means, within a network of client computers, all in the cloud computing and storage is completed, the client belongs to the thin client, similar to the previous diskless workstation, the client does not set up a storage device or less, with low-end CPU is used only for network communications.

VMware Horizon View is vMwarevSphere virtualization platform for enterprise-class desktop virtualization solution that can be deployed directly on vMwarevSphere virtualization platform, greatly integrate existing resources.

VMware Horizon View desktop virtualization environment must use a domain, that is, you must install Microsoft's Active Directory server. In addition, VMware Horizon View desktop virtualization services, and vCenter Server also relies on a separate database support. VMware Horizon View desktop virtualization is a core component of View Composer Server and View Connection Server. The former can quickly deploy thousands of virtual desktops and can save 70\% of storage space. The latter is mainly used to configure the maintenance of desktop virtualization.

Currently, some institutions are still in office or room has some running Windows XP computers that need to gradually upgrade to Windows 7. And now with VMware Horizon View virtual desktops, we can solve this problem by configuring the VMware View desktop. Users can use the View Client Login using 7 even after Windows Windows 10 virtual desktops. And the user's data is still stored on the local hard drive running Windows XP's. After a period of time using the View desktop, you can replace the original host PC to support VMware View thin clients, in order to achieve energy saving purposes (traditional host PC power about $130 \mathrm{~W}$, while the new power of thin clients is less than $20 \mathrm{~W}$ or even only a few W).

\section{VCL Construction Points and Optimization}

This section describes the use Vmware virtualization software to build high availability VCL and operation and maintenance of some of the points and optimization experience.

Rational Allocation Using Vsphere Advanced Features. VmwarevSphere virtualization software provides vMotion (live migration), HA (high availability), DRS (Dynamic Resource Balancing), FT (fault-tolerant) and other advanced features greatly improve the operational efficiency of maintenance work. But improperly configured will affect the normal operation of the overall virtualization platform.

vMotion is the basis of all advanced features. Because when a vMotion operation, the instantaneous data traffic will occupy the full bandwidth, Gigabit and above, a separate network card traffic flow is a must.

HA key is used to calculate the overall good of resources, it is recommended ESXi host load of about $70 \%$. For ESXi hosts less than 10 the number of environments and HA uses two access control policy: "Host Failures Cluster Tolerates" and "as a failover cluster percentage of space reserved resources" can be used. If your ESXi host is greater than the number 10, it is preferable to use the latter strategy. In addition, the important virtual machines set to start high priority are also necessary. For VM Monitoring sensitivity is generally set to medium, to avoid too frequent restart the virtual machine.

VmwarevSphere virtualization software DRS automation is an important part, it can reduce the maintenance burden virtualization management personnel, so this feature has been widely used. If the virtualization platform with the same model ESXi host, it is recommended to use "automatic" mode. Otherwise, you should select "Manual" or "semi-automatic" to prevent the virtual machine configuration higher ESXi hosts cannot automatically migrated to the lower configuration of ESXi hosts. DRS advanced features of the group management is very meaningful function, grouping by a virtual machine, to avoid providing the same service virtual machine (such as 2 Active Directory Server) running on a single ESXi host.

FT is in the so-called two physical machines running the same two virtual machines to achieve low-cost dual-system hot backup function. However, due to technical limitations FT virtual machine can only use one vCPU, its two physical machine must be exactly the same, cannot have virtual drives and other equipment, a lot of restrictions, the effect is really general. After several switching 
FT, FT sometimes backup will not start automatically, sometimes redo FT, FT prompted to configure not compatible with, and restart a virtual machine but can be compatible. It is recommended practice not to use FT.

Design and Implementation of Vcenter Server and Its Optimizing. As vMwarevSphere vCenter Server virtual infrastructure management platform has a very important role. Whether the vCenter Server running on the ESXi host or on a physical server, it is recommended that each vCenter Server configuration 4vCPU, 8GB of memory, while using vCenter Server Heartbeat, vCenter Server servers to achieve redundancy.

The choice of vCenter Server database, the general recommendation is greater than the number of hosts ESXi 5 uses a separate database rather than the embedded database, it avoids VmwareVirtualCenter Server service cannot start automatically issue. If you select a standalone database, it is recommended to select a fairly stable Windows Server 2008 R2 + SQL Server 2008 and do not try to install a combination of configuration and maintenance are more complex Oracle databases.

Construction of High-Performance Network Environment. Whether ESXi host, network storage, virtual machines, and so rely on the network to work properly provide services. For virtualization platform design and implementation staff, high-performance equipment is definitely the first choice, but the project budget is everything. If conditions permit, Gigabit LAN is the preferred virtual machine traffic and storage traffic. Software-based distributed switch to enhance network performance may be negligible, for more than 10 ESXi host data center, using software-based distributed switch can help bring daily management. For VLAN division, it proposed to storage traffic, virtual machine traffic, FT flow divided into different VLAN.

Performance Optimization of Storage. Performance relational storage virtualization to the entire vMwarevSphere speed internet and appropriate optimization is very important. IBM, HP, EMC and other storage devices can provide professional-grade high-capacity, high fault tolerance, real-time synchronization and other storage functions, but its high price is the general enterprises cannot afford. In fact, in addition to professional-grade storage devices, you can use vMware official VSA tool or use Linux (deprecated unstable Windows) to build iSCSI and NFS storage as a storage vMwarevSphere virtualization platform. According to the actual situation, for the next memory read and write is not very frequent in the environment, choose the NFS storage is not much problem, and is relatively simple to configure and maintain. If you are using iSCSI storage environments in the read and write frequently, pay attention ESXi host iSCSI storage traffic binding. In addition, the storage server to make use of SAS hard drives for increased speed, high-capacity SATA hard disk can be stored as a backup server.

Virtual Desktop Optimization. (1) Bandwidth: No matter how much data desktop virtualization to run, it is recommended to use Gigabit Ethernet as an access, the core 10 Gigabit networks.

(2) Maximize the use of high-profile ESXi host to prevent the entire desktop virtualization running slow.

(3) To optimize virtual desktop template. When using vMware Horizon View desktop virtualization, either Windows XP or Windows 7 templates, we need to make the necessary optimization to improve overall performance. These optimizations include: hard disk space allocation recommended that ordinary users are assigned 40GB space can be, and software development user-assigned 60GB-80GB space to meet their requirements; remove the floppy disk drive; removing the CD / DVD drive boot; modify the Windows 7 Desktop Theme "Windows classic"; adjust the default Windows 7 visual effects for the "adjust for best performance"; Turn off system restore; disable communication ports.

\section{Conclusion}

VCL utilization of cloud computing technology, virtualization technology and Internet technology, breaking existing physical computer lab and space constraints, and improve the utilization of computer practice teaching quality of existing computing resources.

Our hospital has purchased new Dell blade servers and has initially achieved a 40 VCL client 
capability, which is the next step in promoting the use of the hospital and the school has laid a solid foundation. Of course, it should be noted VCL itself, there are still some problems. First, the cloud service uses a centralized, easy to produce bottleneck network central node, high network bandwidth requirements; secondly, data security concern. With the rapid updating of computer hardware and software, cloud computing technology, the continuous development of virtualization technology, VCL can fully meet the needs of the campus computer services, it is undoubtedly a room in the future construction of university education as well as information technology and an effective way to development.

\section{References}

[1] Xia Chunqin. Electronics and Software Engineering, Vol. 19 (2013) No 53, p.205-206

[2] Corbin J R. The Art of Distributed Applications: Programming Techniques for Remote Procedure Calls. New York: Springer-Verlag, 1991

[3] Wang Chengwei, Gao Wen, Wang Xingren. Theory, implementation and application of Virtual Reality (VR) technology, Beijing: Tsinghua University Press, 1996

13901054243

孙庆华

北京联合大学机电学院

地址: 北京市朝阳区白家庄西里 12 号

邮编: 100020

\section{暑假邮寄到:}

北京海淀东北旺西路 8 号中关村软件园 1\#楼信息中心 A 座 A306 马媛 收 13901054243 